\title{
Effectiveness of hypoxia-inducible factor prolyl hydroxylase inhibitor roxadustat on renal anemia in non-dialysis-dependent chronic kidney disease: a systematic review and meta-analysis
}

\author{
Linpei Jia ${ }^{1}$, Xingtong Dong ${ }^{1}$, Jingyan Yang ${ }^{2}$, Rufu Jia $^{2}$, Hongliang Zhang ${ }^{3}$ \\ ${ }^{1}$ Department of Nephrology, Xuanwu Hospital, Capital Medical University, Beijing 100053, China; ${ }^{2}$ Central Hospital of Cangzhou, Cangzhou \\ 061001, China; ${ }^{3}$ Department of Life Sciences, the National Natural Science Foundation of China, Beijing 100085, China \\ Contributions: (I) Conception and design: L Jia, R Jia, H Zhang, J Yang; (II) Administrative support: None; (III) Provision of study materials or \\ patients: None; (IV) Collection and assembly of data: L Jia, Q Jia, R Jia; (V) Data analysis and interpretation: L Jia, H Zhang; (VI) Manuscript \\ writing: All authors; (VII) Final approval of manuscript: All authors. \\ Correspondence to: Dr. Linpei Jia. Department of Nephrology, Xuanwu Hospital, Capital Medical University, Changchun Street 45\#, Beijing 100053, \\ China. Email: anny_069@163.com; Dr. Rufu Jia. Central Hospital of Cangzhou, Xinhua Middle Street 201\#, Cangzhou 061001, China. \\ Email: zxyy5688@126.com; Dr. Hongliang Zhang. Department of Life Sciences, the National Natural Science Foundation of China, Shuangqing \\ Road 83\#, Beijing 100085, China. Email: drzhl@hotmail.com.
}

Background: Renal anemia is a severe complication of chronic kidney disease (CKD) and may worsen its prognosis. Roxadustat is the only oral hypoxia-inducible factor prolyl hydroxylase inhibitor (HIF-PHI) that has been proved effective to treat renal anemia. However, effects of roxadustat on non-dialysis-dependent CKD (NDD-CKD) have yet to be supported by evidence-based medicine.

Methods: Based on the databases of PubMed, EMBASE and Web of Science by 12 April 2019 (CRD42019133225), a meta-analysis of randomized controlled trials (RCTs) on roxadustat for treatment of NDD-CKD was conducted. Primary outcomes were parameters of hemoglobin $(\mathrm{Hb})$ and $\mathrm{Hb}$ response. Secondary outcomes were hepcidin, ferritin, total iron binding capacity (TIBC), transferrin saturation (TAST), incidences of diarrhea, adverse events (AEs) and severe adverse events (SAEs). The risk of bias and the quality of evidence were assessed, respectively. Both continuous and binary variables were analyzed by the random effects models. Sensitivity analyses were performed when a significant heterogeneity was observed $\left(\mathrm{P}<0.1\right.$ and $\left.\mathrm{I}^{2}>50 \%\right)$.

Results: Finally, three studies with a total of 214 subjects in the roxadustat group and 80 subjects in the placebo group were enrolled. An increase of $\mathrm{Hb}$ [weighted mean difference $(\mathrm{WMD})=1.22,95 \% \mathrm{CI}$ : 0.95 to 1.49, $\mathrm{P}<0.01$ ], $\mathrm{Hb}$ response [odds ratio $(\mathrm{OR})=27.74,95 \% \mathrm{CI}: 10.18$ to $75.62, \mathrm{P}<0.00001$ ], and TIBC [standard mean difference $(\mathrm{SMD})=1.59,95 \% \mathrm{CI}: 1.17$ to 2.01, $\mathrm{P}<0.00001]$ was found. A decrease of hepcidin (SMD $=-4.46,95 \%$ CI: -5.02 to $-3.89, \mathrm{P}<0.00001)$, ferritin (WMD $=-61.05,95 \%$ CI: -85.70 to $-36.40, \mathrm{P}<0.00001)$ and TAST (WMD $=-6.55,95 \% \mathrm{CI}:-8.82$ to $-4.29, \mathrm{P}<0.00001)$ were noted as well. Analyses of incidence in diarrhea ( $\mathrm{OR}=1.54,95 \% \mathrm{CI}: 0.49$ to $4.79, \mathrm{P}=0.46)$, AEs ( $\mathrm{OR}=1.31,95 \%$ CI: 0.76 to $2.27, \mathrm{P}=0.34)$ and SAEs ( $\mathrm{OR}=1.25,95 \%$ CI: 0.29 to $5.35, \mathrm{P}=0.76$ ) yielded no difference between the roxadustat and the placebo groups. Conclusions: Roxadustat improved renal anemia of NDD-CKD patients by improving Hb and iron metabolism. Oral administration of roxadustat was relatively safe in that roxadustat did not increase the incidence of AEs and SAEs.

Keywords: Roxadustat; chronic kidney disease (CKD); renal anemia; hypoxia-inducible factor prolyl hydroxylase inhibitor (HIF-PHI); meta-analysis

Submitted Jun 25, 2019. Accepted for publication Nov 05, 2019.

doi: 10.21037/atm.2019.12.18

View this article at: http://dx.doi.org/10.21037/atm.2019.12.18 


\section{Introduction}

Renal anemia is one of the most common complications of chronic kidney disease (CKD), which influences the living quality and prognosis of patients (1). Nearly all patients at the advanced stage of CKD suffer from anemia, and a considerable portion of patients are not adequately treated (1). Severe renal anemia may increase the occurrence of cardiovascular events, cognitive impairment and longterm hospitalization, etc. (2).

For CKD patients, fibrosis of kidney tissue reduces the production of erythropoietin (EPO). Lack of EPO further influences the transportation and utilization of the iron metabolism and leads to renal anemia. Thus, iron supplementation and conventional erythropoiesisstimulating agents (ESAs) like recombinant EPO or related analogues are two first-line therapies in clinical practice (3). Although ESAs have been confirmed as an efficient therapy, their possible adverse events (AEs) have raised concern. For example, higher risks of cardiovascular and cerebrovascular events in association with the use of ESAs have been found in patients with hemoglobin $(\mathrm{Hb})>13 \mathrm{~g} / \mathrm{L}$ in various clinical studies $(4,5)$. Thus, the kidney disease improving global outcomes (KDIGO) clinical practice guideline has lowered the target value of $\mathrm{Hb}$ to $11.5-12.5 \mathrm{~g} / \mathrm{L}$ for nondialysis-dependent (NDD) CKD (NDD-CKD) patients (6), which may consequently lower patients' qualities of life and increase the frequency of blood transfusion. Therefore, safe and efficient alternatives remain an unmet need for renal anemia in NDD-CKD patients.

The hypoxia-inducible factor prolyl hydroxylase inhibitors (HIF-PHIs) are being developed as a new therapy for anemia in patients with CKD. Several HIF-PHIs have reached advanced stages of development in either phase II or III clinical trials, such as vadadustat, molidustat and daprodustat (3). Roxadustat, also called FG-4592, is the only HIF-PHI that has approved by the Food and Drug Administration for clinical use (3). Being used in clinical practice in America, China and several other countries, roxadustat negatively regulates the activity of prolyl hydroxylases of HIF and inhibits the degradation of HIF (7). HIF up-regulates the sensitivity of the EPO receptor and improves iron metabolism (8). HIF-PHI increased the level of $\mathrm{Hb}$ in Wistar rats receiving subtotal nephrectomy, while endogenous EPO levels remained within the normal range (9). Several clinical studies have reported clear evidence of efficacy of roxadustat in reversing the decrease of $\mathrm{Hb}$ in NDD-CKD patients $(10,11)$; however, the sample size of these studies is not large enough. It is therefore necessary to reveal the therapeutic effects and safety of roxadustat by an evidence-based method.

In this study, we systematically reviewed randomized controlled trials (RCTs) on roxadustat for treating renal anemia of NDD-CKD patients. A meta-analysis was done to assess the therapeutic effectiveness of roxadustat on $\mathrm{Hb}$, iron metabolism and safety. Gaps in literature were finally highlighted for guiding future follow-up studies.

\section{Methods}

Our study was conducted as per the preferred reporting items for systematic reviews and meta-analyses (PRISMA) statement (Table S1, http://www.prisma-statement. org/). We have registered the study at the International Prospective Register of Systematic Reviews (https://www. crd.york.ac.uk/PROSPERO/, No. CRD42019133225).

\section{Searching strategy}

Entry terms of "chronic kidney disease", "end stage renal disease" and "roxadustat" were combined to search the related articles in electronic medical databases, including PubMed, EMBASE and Web of Science. The searching strategy was described in Supplementary. All publications in English until 12 April 2019 were selected without restriction of origins, countries or article types. Two reviewers screened reference lists of all publications to identify appropriate studies which may be left out in the initial searching.

\section{Inclusion and exclusion criteria}

Two independent authors (L Jia and R Jia) assessed the yielded articles. Initially, titles and abstracts were looked through to identify eligible studies. Then full-texts were evaluated to select studies for inclusion in the meta-analysis. If discrepancies existed, a third researcher (H Zhang) would make the judgment to ensure the accuracy.

The inclusion criteria were: (I) RCTs only, regardless of the design of blindness; (II) adult CKD patients who were diagnosed with renal anemia and who were independent of dialysis; (III) roxadustat for treatment groups and placebo for controls without restriction of dosage or frequency; (IV) available outcomes of $\mathrm{Hb}$, iron metabolism and AEs. If one cohort was reported in several publications, only the article with the largest sample size and the longest duration was 
chosen.

The exclusion criteria were: (I) non-RCT studies, animal experiments, or in vitro studies; (II) non-original studies or case studies, including reviews, conference abstracts and case reports/series; (III) dialysis-dependent CKD patients.

\section{Outcome measures}

The primary outcomes were changes of $\mathrm{Hb}$ and $\mathrm{Hb}$ response. The secondary outcomes were changes of iron metabolism parameters, including hepcidin, ferritin, total iron binding capacity (TIBC) and transferrin saturation (TAST), incidence of diarrhea, incidence of AEs and incidence of severe adverse events (SAEs), following the use of roxadustat. Common drug related AEs included gastrointestinal disorder, headache, dizziness, peripheral edema, hypertension, urinary infection and so forth. SAEs refer to acute kidney injury, heart failure, severe infections, etc.

\section{Data extraction}

Eligible data were extracted from full-texts and supplementary materials by two researchers (Linpei Jia and Xingtong Dong) independently. Occasionally missing data were requested from corresponding authors via Emails. Discrepancies during data extraction were judged by a third researcher (Hongliang Zhang). Data of each trial, including basic information of studies (authors, publication year, countries and study year), demographic data of participants (mean age, sample size, stage of CKD and baseline laboratory parameters), details of roxadustat use (dosage, manufacturer and treatment duration) and each outcome parameters, were recorded.

\section{Quality assessment and summary of findings (SoF)}

The risk of bias of all eligible studies was evaluated by the Cochrane Collaboration's tool according to the following items: random sequence generation, allocation concealment, blinding of patients, blinding of outcome assessment, completeness of outcome data, selective reporting and other bias (12). We also estimated the quality of evidence according to the Grading of Recommendation Assessment (GRADE) method according to the risk of bias, inconsistency, indirectness, imprecision, and publication bias by the GRADEpro GDT 2015 (13). Two independent reviewers (R Jia and J Yang) finished the quality assessment and GRADE. Discrepancies were referred to a third reviewer (H Zhang).

\section{Data pooling and analysis}

Continuous variables were analyzed by the inverse variance method, and discontinuous variables were analyzed by the Mantel-Haenszel method (14). The random effects model was used. Results were expressed as weighted mean difference (WMD) for continuous data. Standard mean difference (SMD) was used for outcomes with different units or larger differences of measurements among studies for continuous data. The odds ratio (OR) was calculated for discontinuous data. The percentage of variability among studies attributable to heterogeneity beyond chance by $\mathrm{I}^{2}$ statistics was calculated. Sensitivity analyses were performed to assess the heterogeneity. RevMan (The Nordic Cochrane Centre, Cochrane Collaboration, Copenhagen, Denmark, Version 5.3) was used to perform the statistical analysis. $\mathrm{P}<0.05$ indicated statistical significance.

\section{Results}

\section{Three trials were finally enrolled}

Initially, 171 articles were searched from databases, including 26 articles from PubMed, 72 articles from EMBASE and 73 articles from Web of Science. One article was identified from references (15). After screening of abstracts and full-texts, 3 studies were finally enrolled (Figure 1) $(10,16,17)$. Data of 314 NDD-CKD patients at stages 1 to 5 were extracted and analyzed (Table 1). The use of EPO was inhibited in all studies to ensure the accuracy. Oral iron should be used continuously if the patients took oral iron before the study. Intravenous iron supplementation was inhibited in two studies and permitted in Akizawa et al.'s study for patients with severe iron insufficiency only (Table 1). Roxadustat in RCTs was manufactured by two independent companies, i.e., Astellas Pharma Inc. (Japan) and FibroGen, Inc. (the United States of America).

\section{Assessment of the risk of bias}

The risk of bias was assessed according to the Cochrane Collaboration's tool (Figure S1 and Table 2). The highest risk appeared in the blinding of outcome assessment because of the single-blinded design in Besarab et al.'s 


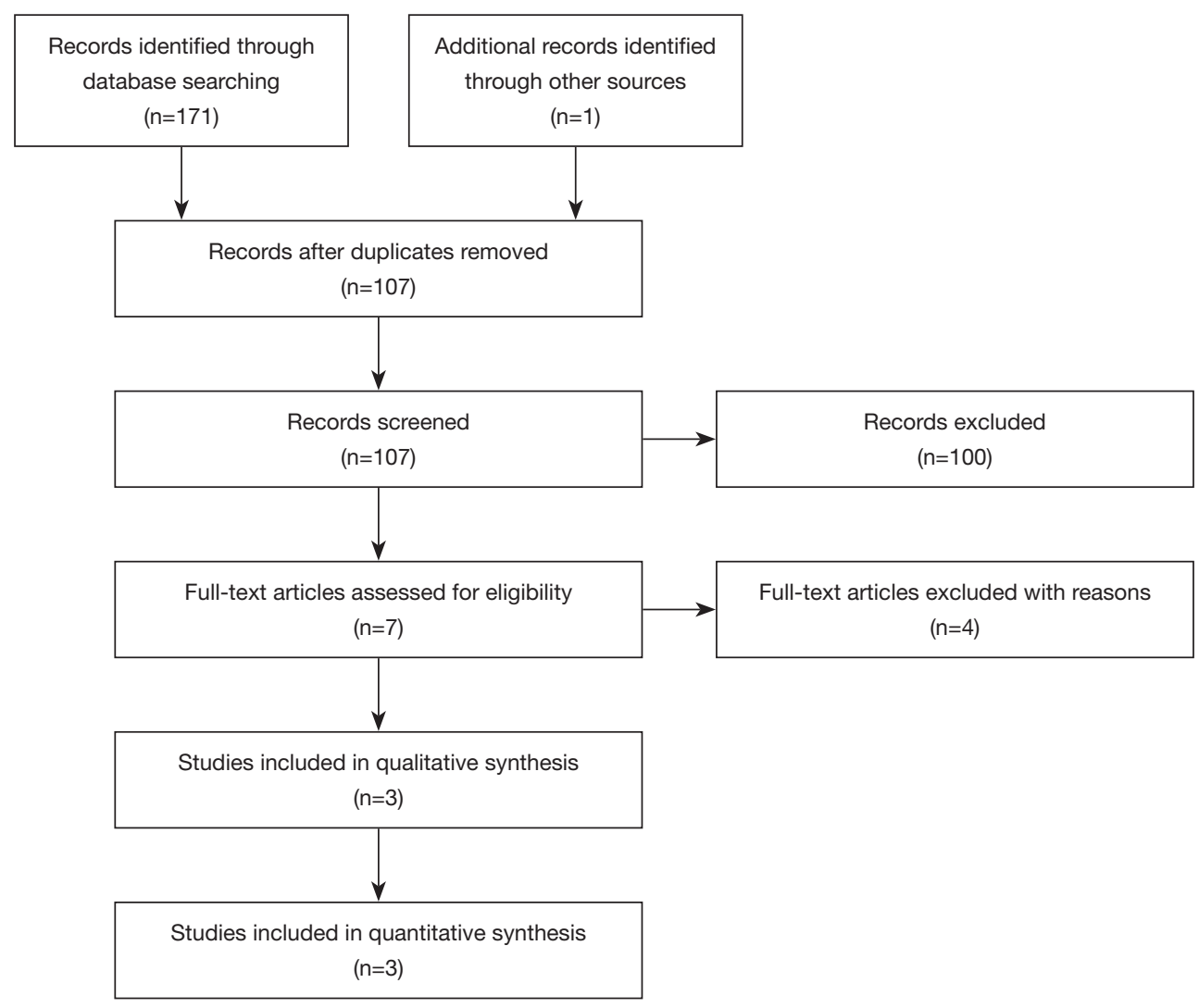

Figure 1 Flow chart of the identification of eligible studies. Initially 171 articles were searched from major databases, including 26 in PubMed, 72 in EMBASE and 73 in Web of Science. One study was identified from references. Duplicate checking was done, and 65 articles were ruled out. Two independent reviewers screened titles and abstracts, and 100 articles were excluded including 69 reviews, 9 animal studies, 9 articles without chronic kidney disease (CKD), 3 articles without roxadustat, and 10 conference abstracts. Then the full-texts of 7 articles were reviewed. Two articles of dialysis dependent CKD patients and one study without placebos were excluded. Because two studies showed results of the same cohort, 3 articles were finally included for meta-analysis.

Table 1 Characteristic studies of meta-analysis

\begin{tabular}{|c|c|c|c|c|c|c|c|c|c|}
\hline Study & Sample size & $\begin{array}{c}\text { Male } \\
\text { ratio \% }\end{array}$ & $\begin{array}{l}\text { Mean } \\
\text { age, y }\end{array}$ & $\begin{array}{l}\text { CKD } \\
\text { stage }\end{array}$ & $\begin{array}{l}\text { Usage } \\
\text { of iron }\end{array}$ & $\begin{array}{l}\text { Use of } \\
\text { EPO }\end{array}$ & Company & $\begin{array}{l}\text { Dosage of } \\
\text { roxadustat }\end{array}$ & $\begin{array}{c}\text { Duration of } \\
\text { treatment }\end{array}$ \\
\hline $\begin{array}{l}\text { Besarab } \\
2015\end{array}$ & $\begin{array}{l}88 \text { subjects/ } \\
28 \text { controls }\end{array}$ & 42.2 & $\begin{array}{c}65.8 \\
{[47-82]}\end{array}$ & 3 to 4 & Oral only & No & FibroGen & $\begin{array}{c}0.7 \mathrm{~g}, 1.0,1.5 \text { and } \\
2.0 \mathrm{mg} / \mathrm{kg} \text { at BIW or } \\
\text { TIW }\end{array}$ & $\begin{array}{c}29 \text { days for BIW } \\
\text { and } 26 \text { days for } \\
\text { TIW }\end{array}$ \\
\hline $\begin{array}{l}\text { Chen } \\
2017\end{array}$ & $\begin{array}{l}61 \text { subjects/ } \\
30 \text { controls }\end{array}$ & 28.6 & $49.7 \pm 13.2$ & 1 to 4 & Oral only & No & FibroGen & $\begin{array}{c}\text { low- }(1.1-1.75 \mathrm{mg} / \mathrm{kg}) \\
\text { and high-dose } \\
(1.50-2.25 \mathrm{mg} / \mathrm{kg}) \mathrm{TIW}\end{array}$ & 8 weeks \\
\hline $\begin{array}{l}\text { Akizawa } \\
2019\end{array}$ & $\begin{array}{l}80 \text { subjects/ } \\
27 \text { controls }\end{array}$ & 46.7 & $63.8 \pm 9.2$ & 2 to 5 & $\begin{array}{l}\text { Oral was allowed } \\
\text { and intravenous iron } \\
\text { was used if TSAT } \\
<5 \% \text { and ferritin } \\
<30 \mathrm{ng} / \mathrm{mL}\end{array}$ & No & Astellas & $\begin{array}{l}50,70 \text { and } 100 \mathrm{mg} \\
\text { TIW in fixed-dose } \\
\text { period, and adjusted } \\
\text { dose in titration period }\end{array}$ & $\begin{array}{c}6 \text { weeks fixed- } \\
\text { dose period and } \\
18 \text { weeks titration } \\
\text { period }\end{array}$ \\
\hline
\end{tabular}

TIW, three times weekly; BIW, two time weekly; EPO, erythropoietin; TAST, transferrin saturation. 
Table 2 The risk of bias of enrolled studies

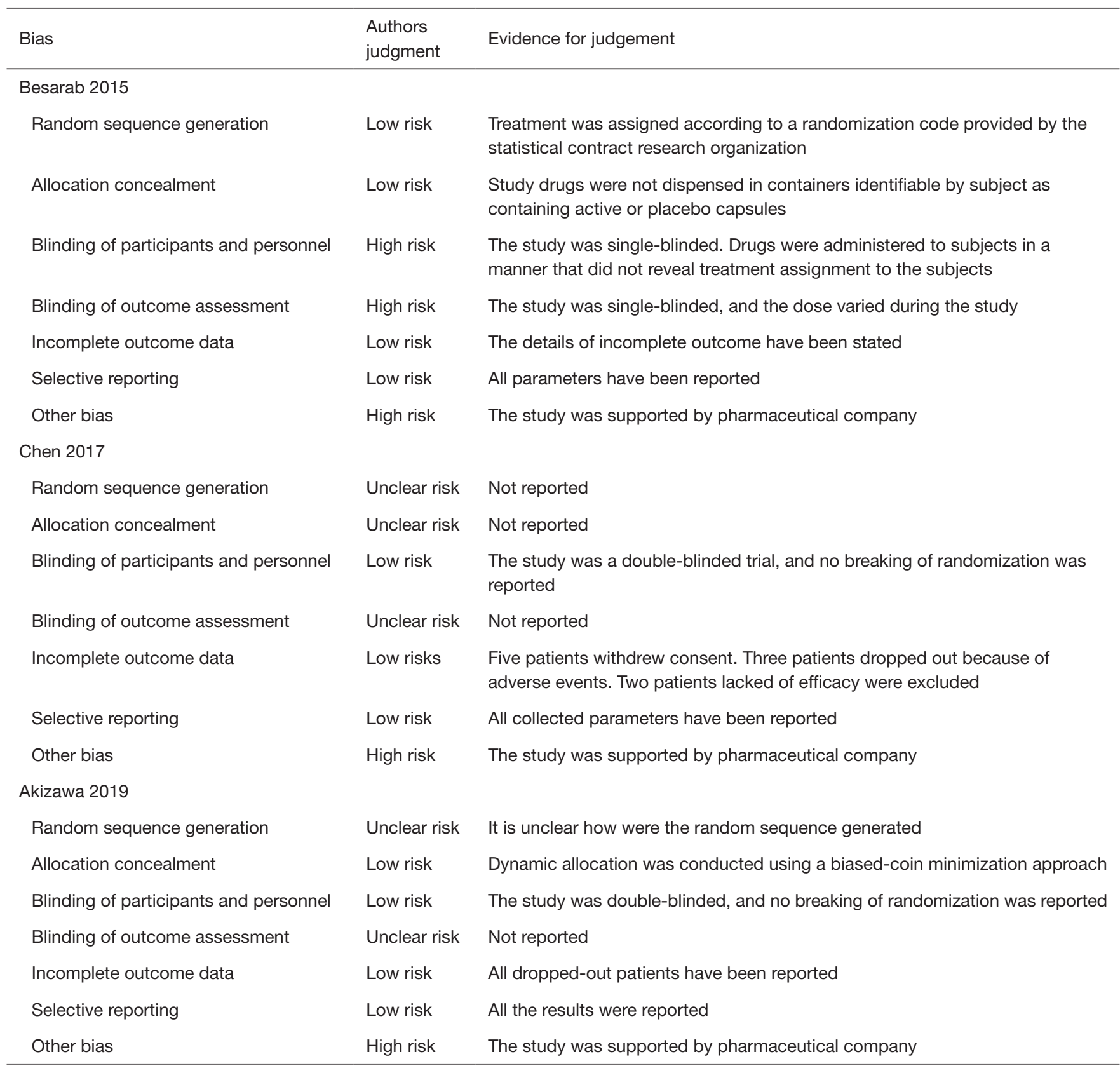

trial. Other biases were assessed as the high risk in all three studies (Figure S1A), because all studies were supported by pharmaceutical companies. The information of random sequence generation and blinding of outcome assessment was missing in Akizawa et al.'s and Chen et al.'s studies (Figure S1B). Description of allocation concealment was missing in Chen et al.'s study. Besarab et al.'s study was in the highest risk among all studies (Figure S1B).

\section{$\triangle F e r r i t i n, A E, S A E$ and incidence of diarrbea were estimated as very-low quality}

We assessed the quality of each outcome, and results were shown in an SoF table (Table 3). The evidence quality of seven outcomes ranged from low to very low. $\triangle \mathrm{Hb}, \mathrm{Hb}$ response, $\triangle$ Hepcidin, $\triangle \mathrm{TIBC}$ and $\triangle \mathrm{TAST}$ were in low qualities, which meant that roxadustat may slightly alter 
Table 3 Summary of findings of roxadustat for renal anemia and AEs of chronic kidney disease (CKD) patients (patient or population: patients with anemia in CKD patients; settings: hospital; intervention: roxadustat)

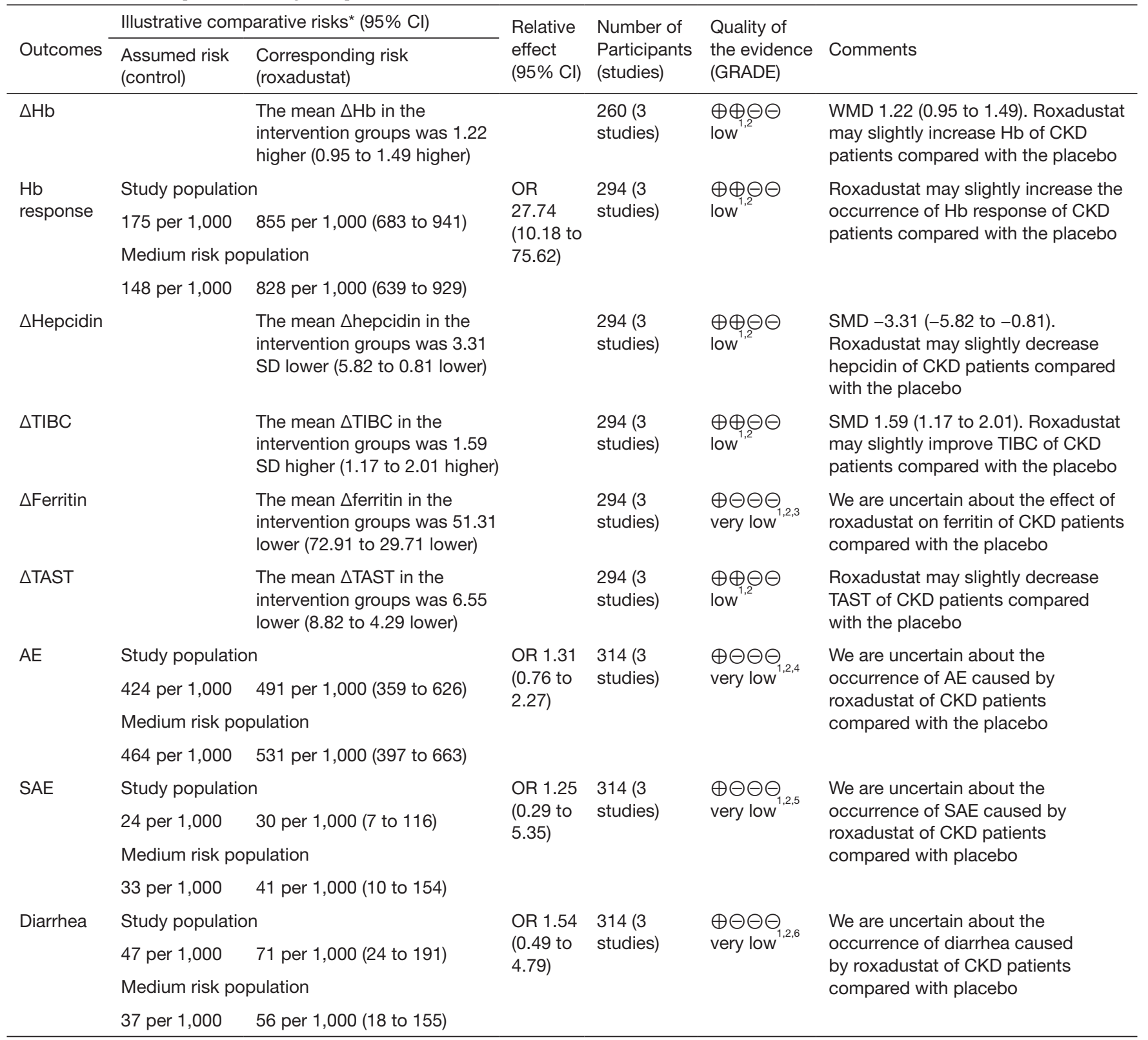

GRADE Working Group grades of evidence high quality: further research is very unlikely to change our confidence in the estimate of effect. Moderate quality: further research is likely to have an important impact on our confidence in the estimate of effect and may change the estimate. Low quality: further research is very likely to have an important impact on our confidence in the estimate of effect and is likely to change the estimate. Very low quality: we are very uncertain about the estimate. *, the basis for the assumed risk (e.g., the median control group risk across studies) is provided in footnotes. The corresponding risk (and its $95 \%$ confidence interval) is based on the assumed risk in the comparison group and the relative effect of the intervention (and its $95 \% \mathrm{Cl}$ ). ${ }^{1}$, imprecise due to the small sample size (less than 300) in all researches. Thus, the evidence quality was downgraded as one level; ${ }^{2}$, some concerns with selected trials conducted by pharmaceuticals companies, thus the evidence quality was downgraded by one level; ${ }^{3}$, one study was inconsistent with the other two in ferritin, thus the quality of evidence was downgraded by one level; ${ }^{4}$, one study was inconsistent with the other two in AEs, thus the quality of evidence was downgraded by one level; ${ }^{5}$, one study was inconsistent with the other two in SAEs, thus the quality of evidence was downgraded by one level; ${ }^{6}$, one study was inconsistent with the other two in diarrhea, thus the quality of evidence was downgraded by one level. Cl, confidence interval; AE, adverse event; CKD, chronic kidney disease; Hb, hemoglobin; OR, odds ration; SAE, severe adverse event; SD, standard deviation; SMD, standard mean difference; TIBC, total iron binding capacity; TAST, transferring saturation; WMD, weighted mean difference. 


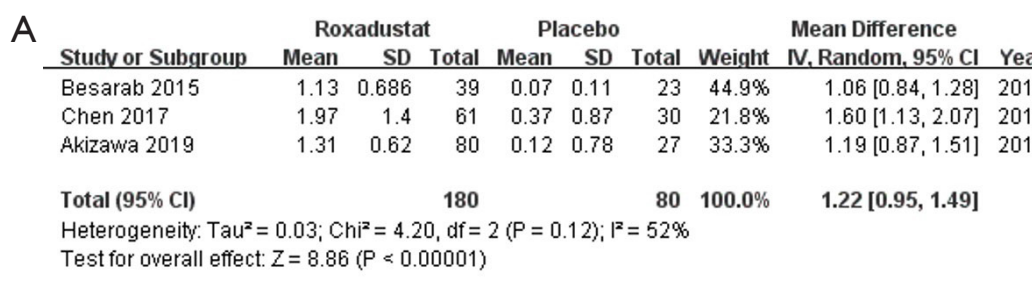

\begin{tabular}{|c|c|c|c|c|c|c|c|c|}
\hline \multirow[t]{8}{*}{ B } & \multirow[b]{2}{*}{ Studv or Subgroup } & \multicolumn{2}{|c|}{ Roxadustat } & \multicolumn{2}{|c|}{ Placebo } & \multicolumn{3}{|c|}{ Odds Ratio } \\
\hline & & Events & Total & Events & Total & Weight & M-H, Random, $95 \% \mathrm{Cl}$ & Year \\
\hline & Besarab 2015 & 53 & 73 & 3 & 23 & $31.7 \%$ & $17.67[4.73,66.00]$ & 2015 \\
\hline & Chen 2017 & 51 & 61 & 7 & 30 & $38.5 \%$ & $16.76[5.67,49.55]$ & 2017 \\
\hline & Akizawa 2019 & 75 & 80 & 4 & 27 & $29.7 \%$ & $86.25[21.37,348.13]$ & 2019 \\
\hline & Total $(95 \% \mathrm{Cl})$ & & 214 & & 80 & $100.0 \%$ & $27.74[10.18,75.62]$ & \\
\hline & Total events & 179 & & 14 & & & & \\
\hline & $\begin{array}{l}\text { Heterogeneity: Tau } \\
\text { Test for overall effec }\end{array}$ & $\begin{array}{l}0.37 ; \mathrm{Chi} \\
\mathrm{Z}=6.49\end{array}$ & $\begin{array}{l}=3.81 \\
P<0.00\end{array}$ & $\begin{array}{l}d f=2(P \\
0001)\end{array}$ & $=0.15$ & 5); $\left.\right|^{2}=479$ & & \\
\hline
\end{tabular}

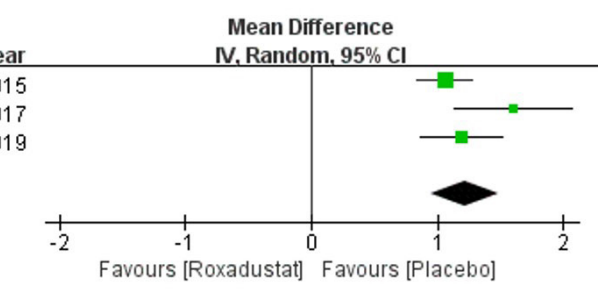

$\Delta \mathrm{Hb}$

\begin{abstract}
Figure 2 Forest plots for comparisons of $\Delta$ hemoglobin $(\mathrm{Hb})$ and $\mathrm{Hb}$ response. In the analysis of $\Delta \mathrm{Hb}$, data of 260 subjects were extracted. A significant increase of $\Delta \mathrm{Hb}$ was found in the roxadustat group (WMD $=1.22,95 \% \mathrm{CI}$ : 0.95 to $1.49, \mathrm{P}<0.00001, \mathrm{~A}$ ). As for the incidence of $\mathrm{Hb}$ response, a significant improvement in the roxadustat group was found as compared with the placebo group (OR =27.74, 95\% CI: 10.18 to $75.62, \mathrm{P}<0.00001, \mathrm{~B})$. WMD, weighted mean difference; $\mathrm{OR}$, odds ratio.
\end{abstract}

these parameters. $\triangle$ Ferritin, $\mathrm{AE}, \mathrm{SAE}$ and occurrence of diarrhea were estimated as very-low qualities, for which we had the limited credibility about the results.

\section{Roxadustat increased the level of $\mathrm{Hb}$ and improved the $\mathrm{Hb}$ response}

A significant increase of $\Delta \mathrm{Hb}$ was found in the roxadustat group as compared with the placebo-controlled patients. The WMD value of $\Delta \mathrm{Hb}$ was 1.22 (95\% CI: 0.95 to 1.49 , $\mathrm{I}^{2}=52 \%, \mathrm{P}<0.00001$, Figure $\left.2 A\right)$. The $\mathrm{Hb}$ response was defined as an increase in $\mathrm{Hb}$ from baseline of $\geq 1 \mathrm{~g} / \mathrm{dL}$ $(10,17,18)$. Subjects who achieved an Hb level of $\geq 1 \mathrm{~g} / \mathrm{dL}$ in Chen et al.'s study and $\geq 10 \mathrm{~g} / \mathrm{dL}$ in Akizawa et al.'s study were also defined as Hb responders. For 294 NDD-CKD subjects, the incidence of $\mathrm{Hb}$ response was significantly improved by roxadustat $(\mathrm{OR}=27.74,95 \% \mathrm{CI}: 10.18$ to 75.62, $\mathrm{I}^{2}=47 \%, \mathrm{P}<0.00001$, Figure $\left.2 B\right)$.

\section{Roxadustat could reduce the level of hepcidin of CKD patients}

Because the mean value of $\Delta$ hepcidin in Besarab et al.'s study $(-224.71 \pm 49.43 \mathrm{mg} / \mathrm{dL})$ was significantly higher than Chen et al.'s $(-37.50 \pm 6.73 \mathrm{mg} / \mathrm{dL})$ and Akizawa et al.'s $(-29.20 \pm$ $24.30 \mathrm{mg} / \mathrm{dL})$ studies, SMD was used in the meta-analysis.
All enrolled studies with 214 subjects in the roxadustat group and 80 subjects in the placebo group reported the data of $\Delta$ hepcidin (SMD $=-3.31,95 \%$ CI: -5.82 to -0.81 , $\mathrm{I}^{2}=98 \%, \mathrm{P}=0.009$, Figure $3 A$ ). The $\mathrm{I}^{2}$ higher than $50 \%$ means an evident heterogeneity, and thus we conducted a sensitivity test accordingly (14). When Besarab et al.'s and Chen $e t$ al.'s studies were ruled out, heterogeneities were still obvious $\left(\mathrm{I}^{2}=98 \%\right.$, Figure $\left.3 B, C\right)$. When the Akizawa et al.'s study was ruled out, the $\mathrm{I}^{2}$ valued $0 \%$ (Figure $3 D$ ). Thus, a significant decrease of hepcidin was found after the treatment of roxadustat (SMD $=-4.46,95 \% \mathrm{CI}:-5.02$ to $-3.89, \mathrm{I}^{2}=0 \%, \mathrm{P}<0.00001$, Figure $\left.3 D\right)$.

\section{Roxadustat decreased serum levels of ferritin}

Changes of ferritin of 214 subjects treated with roxadustat and 80 subjects treated with placebo were available. The meta-analysis of all three studies also showed a great heterogeneity of the serum levels of ferritin (WMD $=-51.31,95 \%$ CI: -72.91 to $-29.71, \mathrm{I}^{2}=63 \%, \mathrm{P}<0.00001$, Figure $4 A$ ). A sensitivity analysis was hence conducted to identify the origin of heterogeneities. After excluding Besarab et al.'s study, the $\mathrm{I}^{2}$ value falls into $44 \%$. Therefore, ferritin was significantly decreased in the roxadustat group as compared with the placebo group (WMD $=-61.05$, 


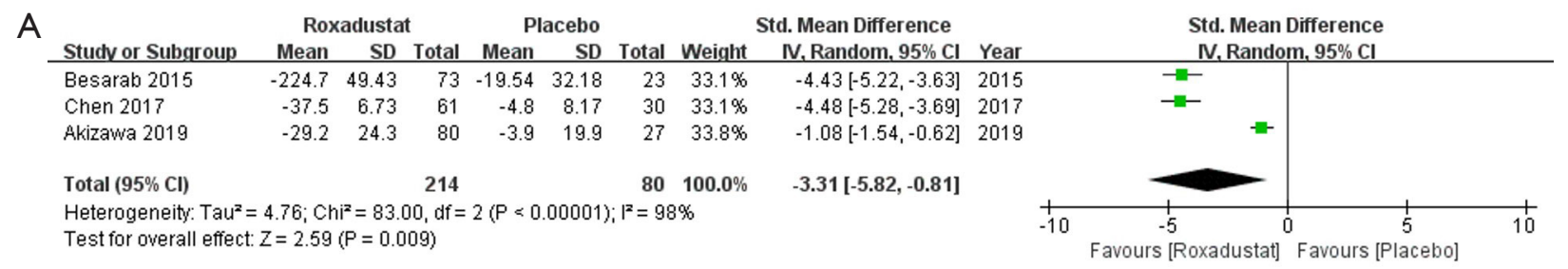

$\Delta$ Hepcidin

\begin{tabular}{|c|c|c|c|c|c|c|c|c|c|}
\hline \multirow[b]{2}{*}{ Studv or Subgroup } & \multicolumn{3}{|c|}{ Roxadustat } & \multicolumn{3}{|c|}{ Placebo } & \multicolumn{3}{|c|}{ Std. Mean Difference } \\
\hline & Mean & SD & Total & Mean & SD & Total & Weight & IV, Random, $95 \% \mathrm{Cl}$ & Year \\
\hline Besarab 2015 & -224.7 & 49.43 & 73 & -19.54 & 32.18 & 23 & $0.0 \%$ & $-4.43[-5.22,-3.63]$ & 2015 \\
\hline Chen 2017 & -37.5 & 6.73 & 61 & -4.8 & 8.17 & 30 & $49.5 \%$ & $-4.48[-5.28,-3.69]$ & 2017 \\
\hline Akizawa 2019 & -29.2 & 24.3 & 80 & -3.9 & 19.9 & 27 & $50.5 \%$ & $-1.08[-1.54,-0.62]$ & 2019 \\
\hline Total $(95 \% \mathrm{Cl})$ & & & 141 & & & 57 & $100.0 \%$ & $-2.77[-6.10,0.57]$ & \\
\hline
\end{tabular}

Test for overall effect: $Z=1.62(P=0.10)$

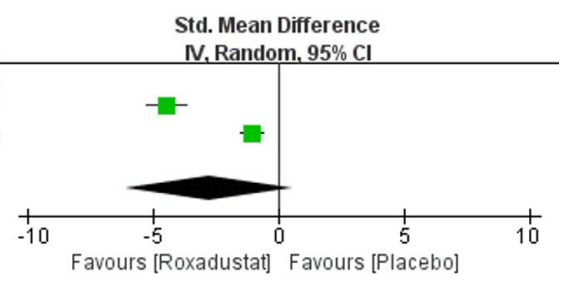

$\Delta$ Hepcidin

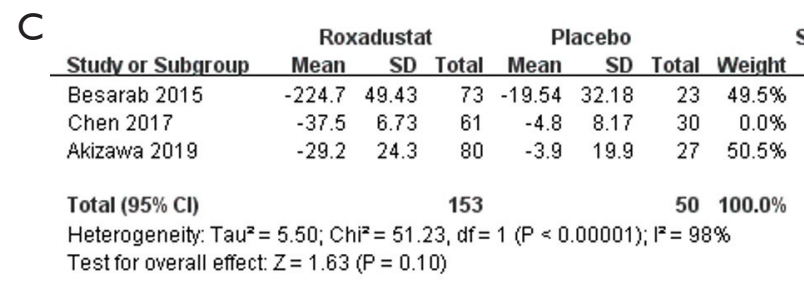
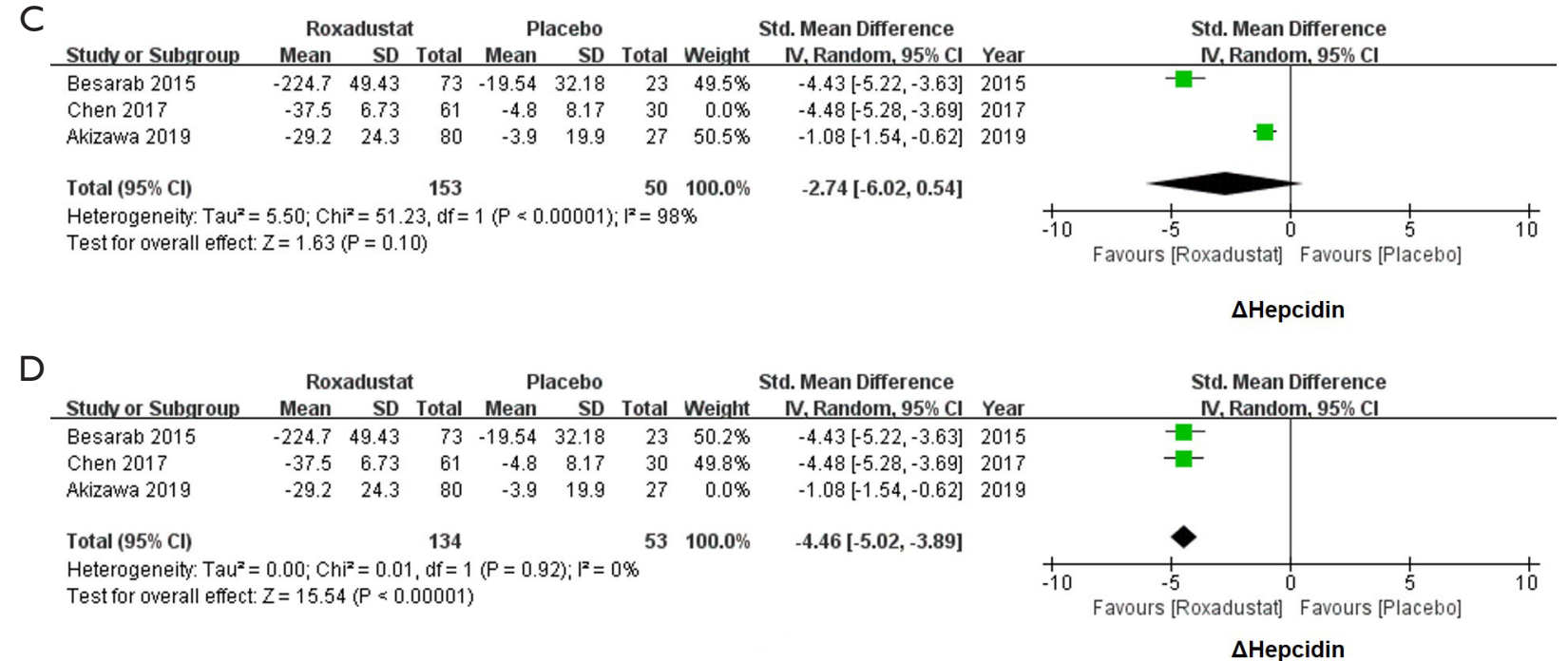

Figure 3 Forest plots for comparisons of $\Delta$ hepcidin. All three enrolled studies reported data of $\Delta$ hepcidin. Meta-analysis yielded a significant heterogeneity (SMD =-3.31, 95\% CI: -5.82 to $-0.81, \mathrm{I}^{2}=98 \%, \mathrm{P}=0.009$, A). Sensitivity analysis was conducted by excluding each study one by one to find out the origin of heterogeneity. After excluding Besarab et al.'s (B) and Chen et al.'s (C) studies, $\mathrm{I}^{2}$ was still higher than $50 \%$. Finally, heterogeneity disappeared after excluding Akizawa et al.'s study, and hepcidin decreased after the treatment of roxadustat (SMD $=-4.46,95 \%$ CI: -5.02 to $\left.-3.89, \mathrm{I}^{2}=0 \%, \mathrm{P}<0.00001, \mathrm{D}\right)$. SMD, standard mean difference.

95\% CI: -85.70 to $-36.40, \mathrm{I}^{2}=44 \%, \mathrm{P}<0.00001$, Figure $\left.4 B\right)$. Heterogeneity did not appear to originate from the Chen $e t$ al.'s or the Akizawa et al.'s studies (Figure 4C,D).

\section{Roxadustat increased TIBC and decreased TAST}

Because the unit of TIBC in 3 studies was inconsistent, SMD was used. By comparing the changes of TIBC in
214 subjects who received roxadustat and 80 subjects who received placebos, an increase of TIBC secondary to roxadustat treatment was significant (SMD $=1.59,95 \%$ CI: 1.17 to $2.01, \mathrm{I}^{2}=52 \%, \mathrm{P}<0.00001$, Figure $5 A$ ). We also analyzed the changes of TAST in the roxadustat and the placebo groups, and a decrease of TAST were significant for subjects treated with roxadustat (WMD $=-6.55,95 \%$ CI: -8.82 to $-4.29, \mathrm{I}^{2}=0 \%, \mathrm{P}<0.00001$, Figure $5 B$ ). 


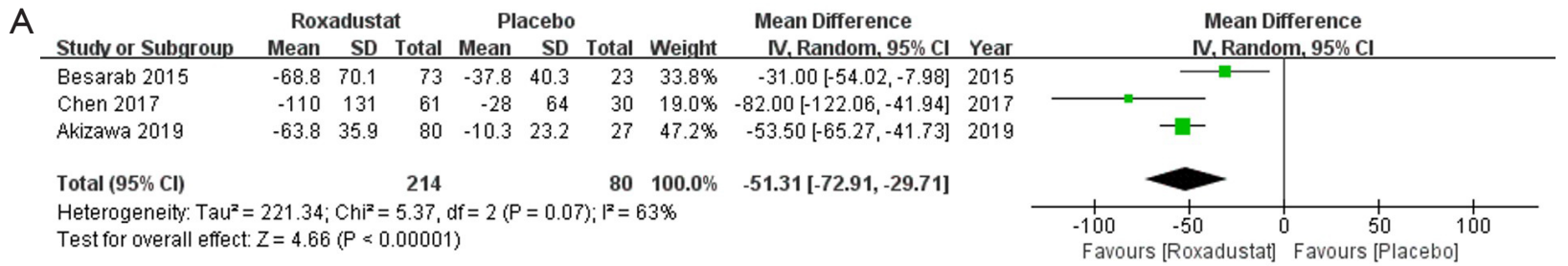
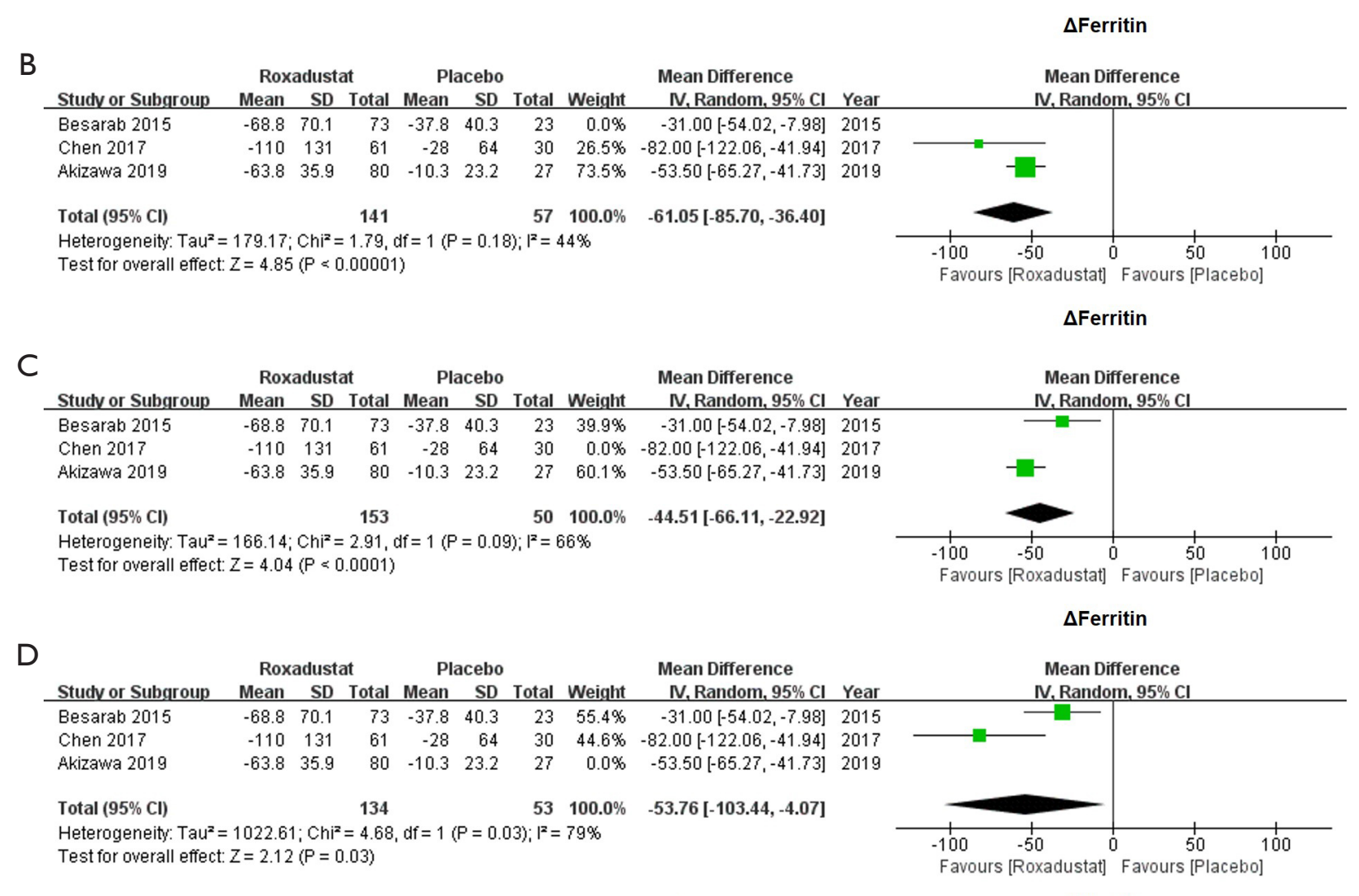

$\Delta$ Ferritin

Figure 4 Forest plots for comparisons of $\Delta$ ferritin. The heterogeneity was found in the meta-analysis of $\Delta$ ferritin (WMD =-51.31, $95 \%$ CI: -72.91 to $\left.-29.71, \mathrm{I}^{2}=63 \%, \mathrm{P}<0.00001, \mathrm{~A}\right)$. After excluding Besarab et al.'s study, a significant decrease of $\Delta$ ferritin was shown (WMD $=-61.05,95 \%$ CI: -85.70 to $\left.-36.40, \mathrm{I}^{2}=44 \%, \mathrm{P}<0.00001, \mathrm{~B}\right)$. In sensitivity analysis, Chen et al.'s (C) and Akizawa et al.'s (D) studies were confirmed not the origin of the heterogeneity. WMD, weighted mean difference.

\section{Roxadustat did not increase AEs and SAEs}

We extracted the data of reported AEs and SAEs of 314 subjects to evaluate whether roxadustat was safe when orally administrated. For all enrolled studies, no significant difference was found in the incidence of diarrhea $(\mathrm{OR}=1.54$, 95\% CI: 0.49 to $4.79, \mathrm{I}^{2}=0 \%, \mathrm{P}=0.46$, Figure $6 A$ ), AEs (OR $=1.31$, 95\% CI: 0.76 to $2.27, \mathrm{I}^{2}=0 \%, \mathrm{P}=0.34$, Figure $6 B)$ and SAEs (OR $=1.25,95 \%$ CI: 0.29 to $5.35, \mathrm{I}^{2}=0 \%$, $\mathrm{P}=0.76$, Figure $6 C$ ) in the roxadustat group compared with the placebo group. Taken together, roxadustat is safe for regular oral use.

\section{Discussion}

In this study, we investigated the therapeutic effectiveness of roxadustat on renal anemia of NDD-CKD patients. Our results showed that roxadustat increased the level of $\mathrm{Hb}$, $\mathrm{Hb}$ response and TIBC. Similarly, roxadustat reduced the levels of hepcidin, ferritin and TAST without increasing 


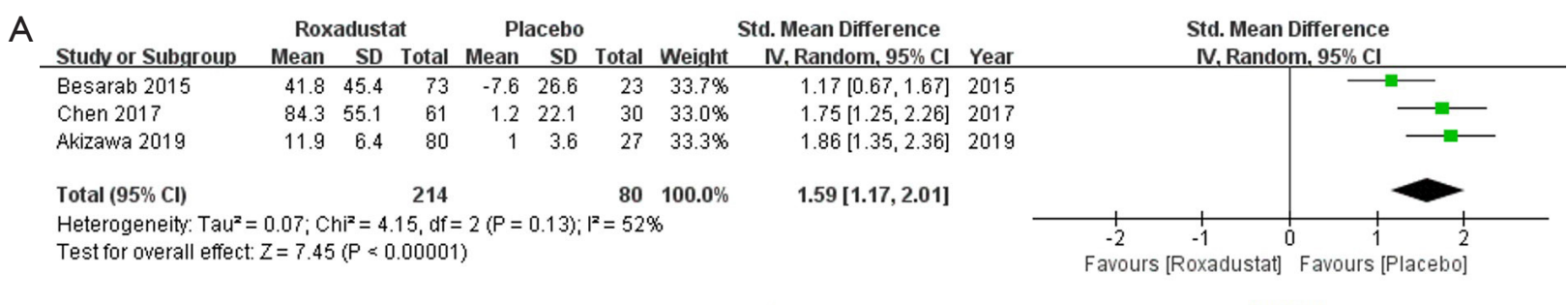

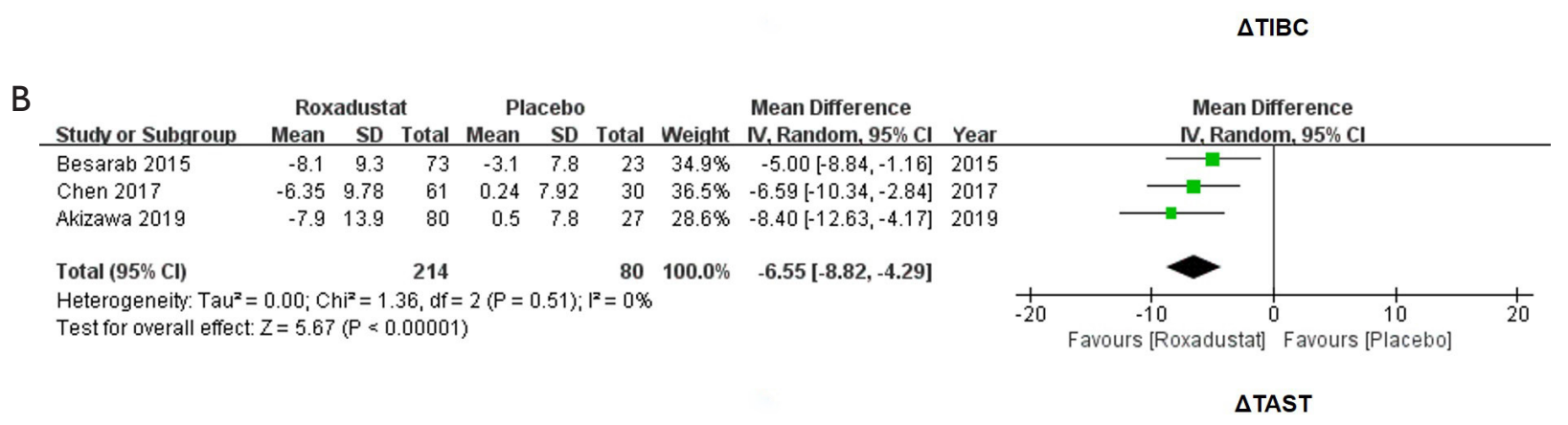

Figure 5 Forest plots for comparisons of $\Delta$ total iron binding capacity (TIBC) and $\Delta$ transferrin saturation (TAST). Meta-analysis of $\Delta$ TIBC showed a significant increase of roxadustat groups compared with placebos (SMD =1.59, 95\% CI: 1.17 to $2.01, \mathrm{I}^{2}=52 \%, \mathrm{P}<0.00001, \mathrm{~A}$ ). $\triangle$ TAST of probiotics groups showed a decrease (WMD $=-6.55,95 \% \mathrm{CI}:-8.82$ to $\left.-4.29, \mathrm{I}^{2}=0 \%, \mathrm{P}<0.00001, \mathrm{~B}\right)$. WMD, weighted mean difference.

AEs and SAEs.

Qualities of published studies were evaluated as low to very-low levels because of the relatively small number of subjects and sponsorship of pharmaceuticals companies. All the three enrolled Phase II studies were well-designed, but important information was still missing in some reports, especially the random sequence generation and discussion of blinding of outcomes, which may lead to the risk of bias. The single-blinded design of Besarab et al.'s study (10) increased the risk of breaking of blindness and may influence the judgment of researchers.

$\mathrm{Hb}$ is recommended as the diagnostic and evaluating indicator of renal anemia. Roxadustat appears to affect $\mathrm{Hb}$ selectively by improving the generation and activity of EPO (19). Several clinical studies have indicated that Roxadustat could significantly increase the level of $\mathrm{Hb}$ in NDD-CKD patients (11). In this meta-analysis, we found that roxadustat not only elevated $\mathrm{Hb}$, but also increased the $\mathrm{Hb}$ response. Patients appeared to be sensitive to roxadustat. Our study confirmed the good curative effect of roxadustat, which is consistent with Zhong et al.'s metaanalysis (3).

Hepcidin, a 25 -amino-acid peptide produced by the hepatocytes, is a key regulator of uptake and release of iron in the tissues to maintain a steady supply of iron to erythron and other tissues while inhibits the entry of iron into systemic circulation. Hepcidin per se is affected by anemia and inflammation (20), which are two major features of CKD. In patients with renal anemia, the level of hepcidin is increased with the deterioration of functional iron deficiency (21). Human studies have shown a reduction of hepcidin after roxadustat treatment in NDD-CKD patients. For CKD patients receiving incident dialysis, the level of hepcidin was significantly decreased after fourweek treatment (22). All these findings were consistent with the result of our meta-analysis. Because roxadustat could promote the production of EPO and further increase the erythroferrone, the suppression of hepcidin may upregulate iron utilization and improve renal anemia $(23,24)$.

The HIF oxygen-sensing pathway has been confirmed as a crucial pathway in maintaining iron hemostasis (25). The significant induction of TIBC and reduction of TAST and ferritin after treatment of roxadustat indicated enhanced iron utilization of NDD-CKD patients. These changes of iron metabolism are not only regulated by the interaction of hepcidin and HIF, but also by other iron-related proteins, such as transferrin receptor, divalent metal transporter 1 , ferropotin 1 and so forth. HIF could directly bind to the hypoxia response elements in mRNAs to regulate expressions of iron-related proteins (26). Recent studies 


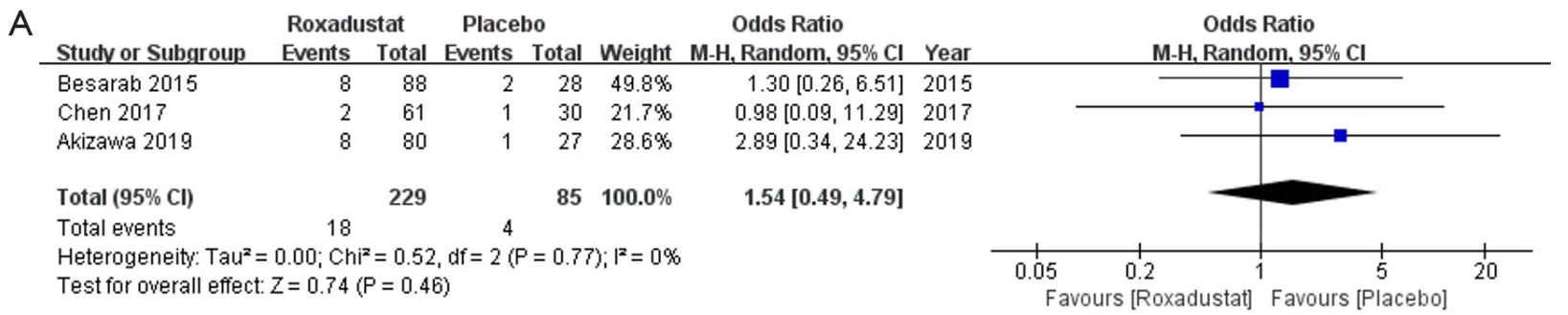

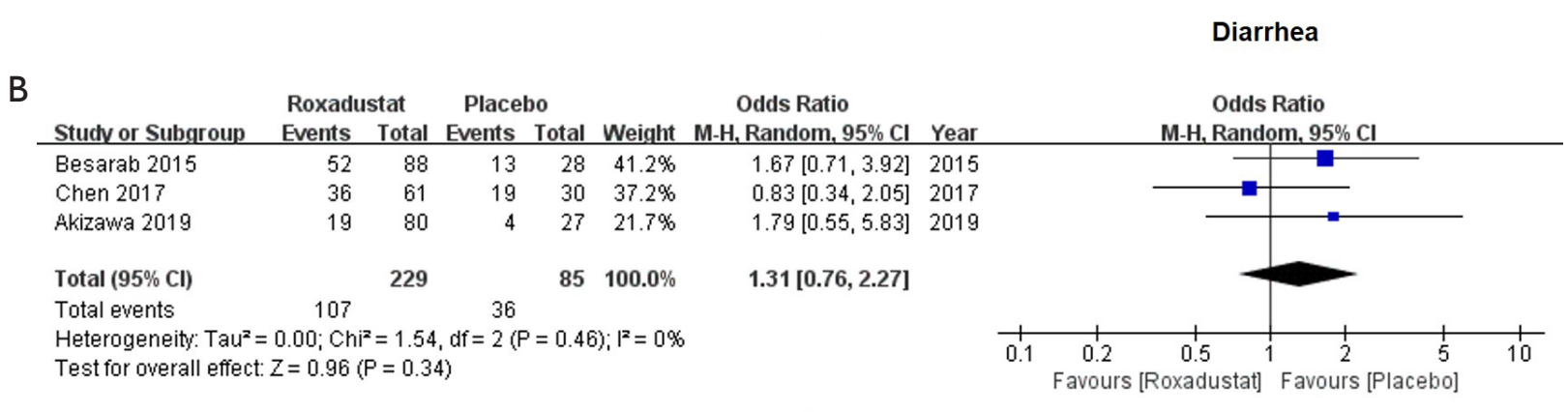

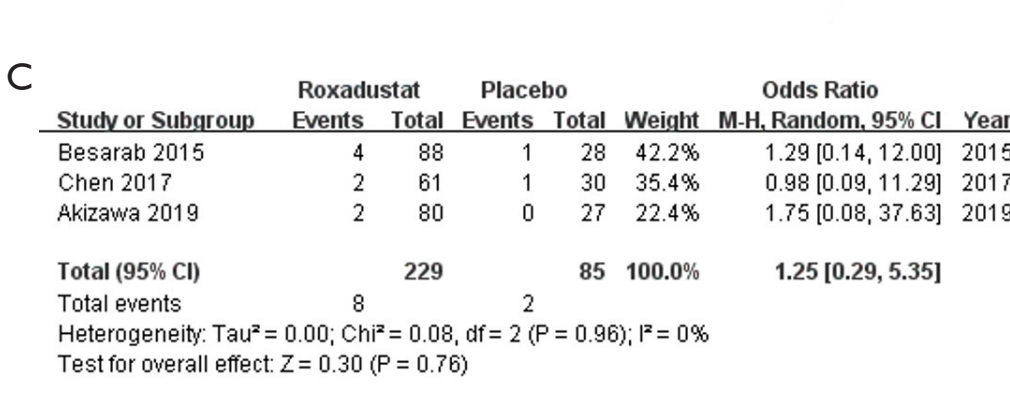

Adverse event

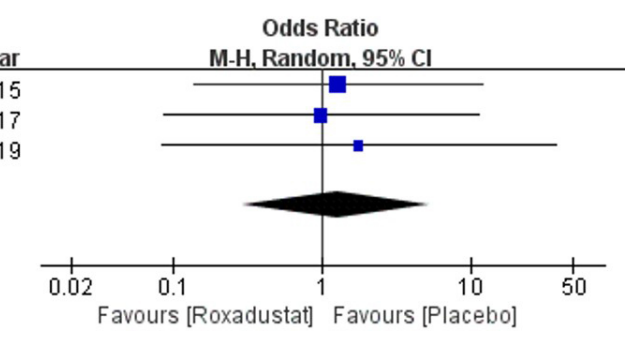

Severe adverse event

Figure 6 Forest plots for comparisons of diarrhea, adverse events and severe adverse events. Our results of meta-analysis showed no significant difference in diarrhea occurrence ( $\mathrm{OR}=1.54,95 \% \mathrm{CI}: 0.49$ to $\left.4.79, \mathrm{I}^{2}=0 \%, \mathrm{P}=0.46, \mathrm{~A}\right)$, adverse events (OR =1.31, $95 \% \mathrm{CI}$ : 0.76 to $\left.2.27, \mathrm{I}^{2}=0 \%, \mathrm{P}=0.34, \mathrm{~B}\right)$ and severe adverse events ( $\mathrm{OR}=1.25,95 \% \mathrm{CI}: 0.29$ to $\left.5.35, \mathrm{I}^{2}=0 \%, \mathrm{P}=0.76, \mathrm{C}\right)$ in comparisons between the roxadustat and the placebo groups. OR, odds ratio.

on HIF- $2 \alpha$ knock-out mice also revealed the critical role of HIF in both iron absorption and iron utilization (27). When it comes to the results of dialysis-dependent patients, no significant changes of ferritin and hepcidin were found after treatment of roxadustat as per Zhong et al.'s meta-analysis (3). HIF stabilizer may be less effective for dialysis-dependent CKD patients than NDD-CKD patients, whereas the underlying mechanism is unknown. According to Provenzano et al.'s study, significant changes of hepcidin and ferritin were noted in dialysis patients after 19-week treatment of roxadustat (28). Thus, the inconsistent conclusions in dialysis and NDD patients may be attributable to short duration and low dose of roxadustat.

Heterogeneities were evident for the serum levels of hepcidin and ferritin. Akizawa et al.'s study contributed to most data discrepancy in the sensitivity analysis of hepcidin. Three possible factors may be attributed to. Firstly, the study by Akizawa et al. defined clear criteria for iron supplement, and they allowed intravenous iron supplement. Secondly, the study by Akizawa et al. used fixed dose in the first six weeks, whereas the other two trials by Chen $e t$ al. and Besarab et al. individualized treatment dose. Third, the manufacturer of roxadustat was different from Akizawa et al.'s study to Chen et al.'s and Besarab et al.'s studies. In the sensitivity analysis of ferritin, Besarab et al.'s study contributed to the data discrepancy, which might be caused by differences of ethnic groups of subjects. Nevertheless, the limited number of enrolled RCTs is the fundamental 
reason. Therefore, more multi-center and clinical trials with large sample size are necessary to provide the evidence of therapeutic effect in renal anemia.

Since HIF and related pathways, especially for the activations of vascular endothelium growth factor (VEGF), have been involved in various biological process, such as cell differentiation, mitochondrial metabolism, and tumor growth (29), changes of HIF expressions would affect functions of multiple organs. AEs and SAEs have been proposed as a great challenge for the clinical use of roxadustat. In all three studies, AEs and SAEs were not significantly increased after the use of roxadustat, which confirmed the safety of roxadustat. However, potential AEs related to the stimulation of VEGFs were not assessed, which might influence the assessment of safety.

Our meta-analysis was the first report focused on the therapeutic effects of roxadustat on renal anemia in NDDCKD patients via an evidence-based method. Only RCTs were included to ensure the quality of our research. Despite these strengths, our study has some limitations. First, the limited number of studies may have an impact on the strength of meta-analysis. Second, because the duration of selected studies was relatively short, the long-term therapeutic effect of roxadustat was unpredictable, and the long-term incidence of AEs and SAEs was unavailable. Third, our work synthesized data from trials with multidose design, thus we may only portray an overview of the effectiveness of roxadustat on renal anemia. An updated meta-analysis with single-dose design of roxadustat is expected. Fourth, all selected studies were supported by pharmaceutical companies, and data analysis might be subject to some bias. Clinical studies of roxadustat conducted by clinical researchers only are expected. Further fully independent clinical trials are warranted to provide high-quality evidence of the efficacy and safety of roxadustat.

\section{Conclusions}

Our meta-analysis provided the evidence that roxadustat could improve the level of $\mathrm{Hb}$ and iron metabolism of NDD-CKD patients. Roxadustat is also safe as the oral therapy for renal anemia in NDD-CKD patients.

\section{Acknowledgments}

Funding: The study was supported by grants from Scientific Research Found of Capital Medical University
(PYZ2018054) to L Jia and Wu Jieping Medical Foundation Clinical Research Funding (No. 320.6750.16050) to R Jia.

\section{Footnote}

Conflicts of Interest: The authors have no conflicts of interest to declare.

Ethical Statement: The authors are accountable for all aspects of the work in ensuring that questions related to the accuracy or integrity of any part of the work are appropriately investigated and resolved.

\section{References}

1. Sugahara M, Tanaka T, Nangaku M. Prolyl hydroxylase domain inhibitors as a novel therapeutic approach against anemia in chronic kidney disease. Kidney Int 2017;92:306-12.

2. Pergola PE, Spinowitz BS, Hartman CS, et al. Vadadustat, a novel oral HIF stabilizer, provides effective anemia treatment in nondialysis-dependent chronic kidney disease. Kidney Int 2016;90:1115-22.

3. Zhong H, Zhou T, Li H, et al. The role of hypoxiainducible factor stabilizers in the treatment of anemia in patients with chronic kidney disease. Drug Des Devel Ther 2018;12:3003-11.

4. Singh AK, Szczech L, Tang KL, et al. Correction of anemia with epoetin alfa in chronic kidney disease. N Engl J Med 2006;355:2085-98.

5. Skali H, Parving HH, Parfrey PS, et al. Stroke in patients with type 2 diabetes mellitus, chronic kidney disease, and anemia treated with Darbepoetin Alfa: the trial to reduce cardiovascular events with Aranesp therapy (TREAT) experience. Circulation 2011;124:2903-8.

6. Drüeke TB, Parfrey PS. Summary of the KDIGO guideline on anemia and comment: reading between the (guide)line(s). Kidney Int 2012;82:952-60.

7. Wyatt CM, Drueke TB. HIF stabilization by prolyl hydroxylase inhibitors for the treatment of anemia in chronic kidney disease. Kidney Int 2016;90:923-5.

8. Maxwell PH, Eckardt KU. HIF prolyl hydroxylase inhibitors for the treatment of renal anaemia and beyond. Nat Rev Nephrol 2016;12:157-68.

9. Flamme I, Oehme F, Ellinghaus P, et al. Mimicking hypoxia to treat anemia: HIF-stabilizer BAY 85-3934 (Molidustat) stimulates erythropoietin production without hypertensive effects. PLoS One 2014;9:e111838. 
10. Besarab A, Provenzano R, Hertel J, et al. Randomized placebo-controlled dose-ranging and pharmacodynamics study of roxadustat (FG-4592) to treat anemia in nondialysis-dependent chronic kidney disease (NDD-CKD) patients. Nephrol Dial Transplant 2015;30:1665-73.

11. Provenzano R, Besarab A, Sun CH, et al. Oral HypoxiaInducible Factor Prolyl Hydroxylase Inhibitor Roxadustat (FG-4592) for the Treatment of Anemia in Patients with CKD. Clin J Am Soc Nephrol 2016;11:982-91.

12. Higgins JP, Altman DG, Gotzsche PC, et al. The Cochrane Collaboration's tool for assessing risk of bias in randomised trials. BMJ 2011;343:d5928.

13. Holger J Schünemann, Andrew D Oxman, Gunn E Vist, et al. Interpreting results and drawing conclusions. In: Julian PT Higgins, Green S, editors. Cochrane Handbook for Systematic Reviews of Intervention. Available online: www.cochranehandbook.org. 2011.

14. Higgins JPT, Green S. Cochrane Handbook for Systematic Reviews of Interventions Version 5.1.0. The Cochrane Collaboration, 2011.

15. Yueheng R. Therapeutic Effect of Stable HypoxiaInducible Factor on Anemia of Chronic Kidney Disease and its Mechanism of Regulating Related Target Genes. Shanghai: Fudan University, 2013.

16. Akizawa T, Iwasaki M, Otsuka T, et al. Roxadustat Treatment of Chronic Kidney Disease-Associated Anemia in Japanese Patients Not on Dialysis: A Phase 2, Randomized, Double-Blind, Placebo-Controlled Trial. Adv Ther 2019;36:1438-54.

17. Chen N, Qian J, Chen J, et al. Phase 2 studies of oral hypoxia-inducible factor prolyl hydroxylase inhibitor FG4592 for treatment of anemia in China. Nephrol Dial Transplant 2017;32:1373-86.

18. Akizawa T, Iwasaki M, Otsuka T, et al. Roxadustat Treatment of Chronic Kidney Disease-Associated Anemia in Japanese Patients Not on Dialysis: A Phase 2, Randomized, Double-Blind, Placebo-Controlled Trial. Adv Ther 2019;36:1438-54.

19. Gupta N, Wish JB. Hypoxia-Inducible Factor Prolyl

Cite this article as: Jia L, Dong X, Yang J, Jia R, Zhang H. Effectiveness of hypoxia-inducible factor prolyl hydroxylase inhibitor roxadustat on renal anemia in non-dialysis-dependent chronic kidney disease: a systematic review and meta-analysis. Ann Transl Med 2019;7(23):720. doi: 10.21037/atm.2019.12.18
Hydroxylase Inhibitors: A Potential New Treatment for Anemia in Patients With CKD. Am J Kidney Dis 2017;69:815-26.

20. Malyszko J, Malyszko JS, Matuszkiewicz-Rowinska J. Hepcidin as a therapeutic target for anemia and inflammation associated with chronic kidney disease. Expert Opin Ther Targets 2019;23:407-21.

21. Nemeth E, Valore EV, Territo M, et al. Hepcidin, a putative mediator of anemia of inflammation, is a type II acute-phase protein. Blood 2003;101:2461-3.

22. Besarab A, Chernyavskaya E, Motylev I, et al. Roxadustat (FG-4592): Correction of Anemia in Incident Dialysis Patients. J Am Soc Nephrol 2016;27:1225-33.

23. Liu Q, Davidoff O, Niss K, et al. Hypoxia-inducible factor regulates hepcidin via erythropoietin-induced erythropoiesis. J Clin Invest 2012;122:4635-44.

24. Hasegawa S, Tanaka T, Nangaku M. Hypoxia-inducible factor stabilizers for treating anemia of chronic kidney disease. Curr Opin Nephrol Hypertens 2018;27:331-8.

25. Anderson ER, Taylor M, Xue X, et al. Intestinal HIF2alpha promotes tissue-iron accumulation in disorders of iron overload with anemia. Proc Natl Acad Sci U S A 2013;110:E4922-30.

26. Tacchini L, Bianchi L, Bernelli-Zazzera A, et al. Transferrin receptor induction by hypoxia. HIF-1mediated transcriptional activation and cell-specific posttranscriptional regulation. J Biol Chem 1999;274:24142-6.

27. Anderson ER, Xue X, Shah YM. Intestinal hypoxiainducible factor-2alpha (HIF-2alpha) is critical for efficient erythropoiesis. J Biol Chem 2011;286:19533-40.

28. Provenzano R, Besarab A, Wright S, et al. Roxadustat (FG-4592) Versus Epoetin Alfa for Anemia in Patients Receiving Maintenance Hemodialysis: A Phase 2, Randomized, 6- to 19-Week, Open-Label, ActiveComparator, Dose-Ranging, Safety and Exploratory Efficacy Study. Am J Kidney Dis 2016;67:912-24.

29. Semenza GL. HIF-1 and mechanisms of hypoxia sensing. Curr Opin Cell Biol 2001;13:167-71. 


\section{Searching strategies of PubMed, EMBASE and Cochrane Library}

\section{For PubMed}

\#1 1 ((()((()(()((()((("Renal Insufficiency, Chronic" [Mesh]) OR Chronic Renal Insufficiencies) OR Renal Insufficiencies, Chronic) OR Chronic Renal Insufficiency) OR Kidney Insufficiency, Chronic) OR Chronic Kidney Insufficiency) OR Chronic Kidney Insufficiencies) OR Kidney Insufficiencies, Chronic) OR Chronic Kidney Diseases) OR Chronic Kidney Disease) OR Disease, Chronic Kidney) OR Diseases, Chronic Kidney) OR Kidney Disease, Chronic) OR Kidney Diseases, Chronic) OR Chronic Renal Diseases) OR Chronic Renal Disease) OR Disease, Chronic Renal) OR Diseases, Chronic Renal) OR Renal Disease, Chronic) OR Renal Diseases, Chronic

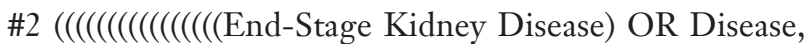
End-Stage Kidney) OR End Stage Kidney Disease) OR Kidney Disease, End-Stage) OR Chronic Kidney Failure) OR End-Stage Renal Disease) OR Disease, End-Stage Renal) OR End Stage Renal Disease) OR Renal Disease, End-Stage) OR Renal Disease, End Stage) OR Renal Failure, End-Stage) OR End-Stage Renal Failure) OR Renal Failure, End Stage) OR Renal Failure, Chronic) OR Chronic Renal Failure) OR ESRD) OR "Kidney Failure, Chronic"[Mesh]

\#3 \#1 OR \#2

\#4 (“FG-4592" [Supplementary Concept]) OR Roxadustat

\#5 \#3 AND \#4

\section{For EMBASE}

\#1 'chronic kidney failure'/exp OR 'chronic kidney failure' \#2 'renal insufficiency, chronic'/exp OR 'renal insufficiency, chronic' OR 'chronic renal insufficiencies' OR 'renal insufficiencies, chronic' OR 'chronic renal insufficiency'/exp OR 'chronic renal insufficiency' OR' kidney insufficiency, chronic' OR 'chronic kidney insufficiency'/exp OR 'chronic kidney insufficiency' OR 'chronic kidney insufficiencies' OR 'kidney insufficiencies, chronic' OR 'chronic kidney diseases' OR 'chronic kidney disease'/exp OR 'chronic kidney disease' OR 'disease, chronic kidney' OR 'diseases, chronic kidney' OR 'kidney disease, chronic'/exp OR 'kidney disease, chronic' OR 'kidney diseases, chronic' OR 'chronic renal diseases' OR 'chronic renal disease'/exp OR 'chronic renal disease' OR 'disease, chronic renal' OR 'diseases, chronic renal' OR 'renal disease, chronic' OR 'renal diseases, chronic'

\#3 \#1 OR \#2

\#4 'end stage renal disease'/exp

\#5 'kidney failure, chronic'/exp OR 'kidney failure, chronic' OR 'end-stage kidney disease'/exp OR 'end-stage kidney disease' OR 'disease, end-stage kidney' OR 'end stage kidney disease'/exp OR 'end stage kidney disease' OR 'kidney disease, end-stage' OR 'chronic kidney failure'/exp OR 'chronic kidney failure' OR 'end-stage renal disease'/ exp OR 'end-stage renal disease' OR 'disease, end-stage renal' OR 'end stage renal disease'/exp OR 'end stage renal disease' OR 'renal disease, end-stage' OR 'renal disease, end stage' OR 'renal failure, end-stage' OR 'end-stage renal failure'/exp OR 'end-stage renal failure' OR 'renal failure, end stage' OR 'renal failure, chronic' OR 'chronic renal failure'/exp OR 'chronic renal failure' OR 'esrd'/exp OR 'esrd'

\#6 \#4 OR \#5

\#7 \#3 OR \#6

\#8 'fg 4592'/exp OR 'fg 4592' OR 'roxadustat'

\#9 \#7 AND \#8

\section{For Web of Science}

\#1 TS = (Chronic Renal Insufficiencies OR Chronic Renal Insufficiency OR Chronic Kidney Insufficiency OR Chronic Kidney Insufficiencies OR Chronic Kidney Diseases OR Chronic Kidney Disease OR Chronic Renal Diseases OR Chronic Renal Disease)

\#2 TS = (End-Stage Kidney Disease OR End Stage Kidney Disease OR Chronic Kidney Failure OR End-Stage Renal Disease OR End Stage Renal Disease OR End-Stage Renal Failure OR Chronic Renal Failure OR ESRD) \#3 \#1 OR \#2 \#4 $\mathrm{TS}=(\mathrm{FG}-4592$ OR FG 4592 OR roxadustat $)$ 
Table S1 Preferred reporting items for systematic reviews and meta-analyses (PRISMA) checklist

\begin{tabular}{|c|c|c|c|}
\hline Section/topic & \# & Checklist item & Reported on page \# \\
\hline \multicolumn{4}{|l|}{ Title } \\
\hline Title & 1 & Identify the report as a systematic review, meta-analysis, or both & P.1 \\
\hline \multicolumn{4}{|l|}{ Abstract } \\
\hline $\begin{array}{l}\text { Structured } \\
\text { summary }\end{array}$ & 2 & $\begin{array}{l}\text { Provide a structured summary including, as applicable: background; objectives; data sources; study eligibility } \\
\text { criteria, participants, and interventions; study appraisal and synthesis methods; results; limitations; conclusions } \\
\text { and implications of key findings; systematic review registration number }\end{array}$ & P.2-3 \\
\hline \multicolumn{4}{|l|}{ Introduction } \\
\hline Rationale & 3 & Describe the rationale for the review in the context of what is already known & P.4-5 \\
\hline Objectives & 4 & $\begin{array}{l}\text { Provide an explicit statement of questions being addressed with reference to participants, interventions, } \\
\text { comparisons, outcomes, and study design (PICOS) }\end{array}$ & P.5 \\
\hline \multicolumn{4}{|l|}{ Methods } \\
\hline $\begin{array}{l}\text { Protocol and } \\
\text { registration }\end{array}$ & 5 & $\begin{array}{l}\text { Indicate if a review protocol exists, if and where it can be accessed (e.g., web address), and, if available, provide } \\
\text { registration information including registration number }\end{array}$ & P.5 \\
\hline Eligibility criteria & 6 & $\begin{array}{l}\text { Specify study characteristics (e.g., PICOS, length of follow-up) and report characteristics (e.g., years considered, } \\
\text { language, publication status) used as criteria for eligibility, giving rationale }\end{array}$ & P.6 \\
\hline Information sources & 7 & $\begin{array}{l}\text { Describe all information sources (e.g., databases with dates of coverage, contact with study authors to identify } \\
\text { additional studies) in the search and date last searched }\end{array}$ & P.6 \\
\hline Search & 8 & $\begin{array}{l}\text { Present full electronic search strategy for at least one database, including any limits used, such that it could be } \\
\text { repeated }\end{array}$ & Table S1 \\
\hline Study selection & 9 & $\begin{array}{l}\text { State the process for selecting studies (i.e., screening, eligibility, included in systematic review, and, if applicable, } \\
\text { included in the meta-analysis) }\end{array}$ & P.6 \\
\hline $\begin{array}{l}\text { Data collection } \\
\text { process }\end{array}$ & 10 & $\begin{array}{l}\text { Describe method of data extraction from reports (e.g., piloted forms, independently, in duplicate) and any } \\
\text { processes for obtaining and confirming data from investigators }\end{array}$ & P.7 \\
\hline Data items & 11 & $\begin{array}{l}\text { List and define all variables for which data were sought (e.g., PICOS, funding sources) and any assumptions and } \\
\text { simplifications made }\end{array}$ & P.7 \\
\hline $\begin{array}{l}\text { Risk of bias in } \\
\text { individual studies }\end{array}$ & 12 & $\begin{array}{l}\text { Describe methods used for assessing risk of bias of individual studies (including specification of whether this was } \\
\text { done at the study or outcome level), and how this information is to be used in any data synthesis }\end{array}$ & P.7 \\
\hline Summary measures & 13 & State the principal summary measures (e.g., risk ratio, difference in means) & P.8 \\
\hline Synthesis of results & 14 & $\begin{array}{l}\text { Describe the methods of handling data and combining results of studies, if done, including measures of } \\
\left.\text { consistency (e.g., }\left.\right|^{2}\right) \text { for each meta-analysis }\end{array}$ & P.8 \\
\hline $\begin{array}{l}\text { Risk of bias across } \\
\text { studies }\end{array}$ & 15 & $\begin{array}{l}\text { Specify any assessment of risk of bias that may affect the cumulative evidence (e.g., publication bias, selective } \\
\text { reporting within studies) }\end{array}$ & P.7 \\
\hline Additional analyses & 16 & $\begin{array}{l}\text { Describe methods of additional analyses (e.g., sensitivity or subgroup analyses, meta-regression), if done, } \\
\text { indicating which were pre-specified }\end{array}$ & P.8 \\
\hline \multicolumn{4}{|l|}{ Results } \\
\hline Study selection & 17 & $\begin{array}{l}\text { Give numbers of studies screened, assessed for eligibility, and included in the review, with reasons for exclusions } \\
\text { at each stage, ideally with a flow diagram }\end{array}$ & P.8 and Figure 1 \\
\hline $\begin{array}{l}\text { Study } \\
\text { characteristics }\end{array}$ & 18 & $\begin{array}{l}\text { For each study, present characteristics for which data were extracted (e.g., study size, PICOS, follow-up period) } \\
\text { and provide the citations }\end{array}$ & Table 1 \\
\hline $\begin{array}{l}\text { Risk of bias within } \\
\text { studies }\end{array}$ & 19 & Present data on risk of bias of each study and, if available, any outcome level assessment (see item 12) & $\begin{array}{l}\text { P.9, Table } 3 \text { and } \\
\text { Figure } S 1\end{array}$ \\
\hline $\begin{array}{l}\text { Results of individual } \\
\text { studies }\end{array}$ & 20 & $\begin{array}{l}\text { For all outcomes considered (benefits or harms), present, for each study: (I) simple summary data for each } \\
\text { intervention group; (II) effect estimates and confidence intervals, ideally with a forest plot }\end{array}$ & Figures 2-5 \\
\hline Synthesis of results & 21 & Present results of each meta-analysis done, including confidence intervals and measures of consistency & P.9-10 \\
\hline $\begin{array}{l}\text { Risk of bias across } \\
\text { studies }\end{array}$ & 22 & Present results of any assessment of risk of bias across studies (see item 15) & Figure S1 \\
\hline Additional analysis & 23 & Give results of additional analyses, if done (e.g., sensitivity or subgroup analyses, meta-regression (see item 16) & P.9-10 \\
\hline \multicolumn{4}{|l|}{ Discussion } \\
\hline $\begin{array}{l}\text { Summary of } \\
\text { evidence }\end{array}$ & 24 & $\begin{array}{l}\text { Summarize the main findings including the strength of evidence for each main outcome; consider their relevance } \\
\text { to key groups (e.g., healthcare providers, users, and policy makers) }\end{array}$ & P.11 \\
\hline Limitations & 25 & $\begin{array}{l}\text { Discuss limitations at study and outcome level (e.g., risk of bias), and at review-level (e.g., incomplete retrieval of } \\
\text { identified research, reporting bias) }\end{array}$ & P.11, 13 and 14 \\
\hline Conclusions & 26 & Provide a general interpretation of the results in the context of other evidence, and implications for future research & P.14 \\
\hline \multicolumn{4}{|l|}{ Funding } \\
\hline Funding & 27 & $\begin{array}{l}\text { Describe sources of funding for the systematic review and other support (e.g., supply of data); role of funders for } \\
\text { the systematic review }\end{array}$ & Acknowledgments \\
\hline
\end{tabular}



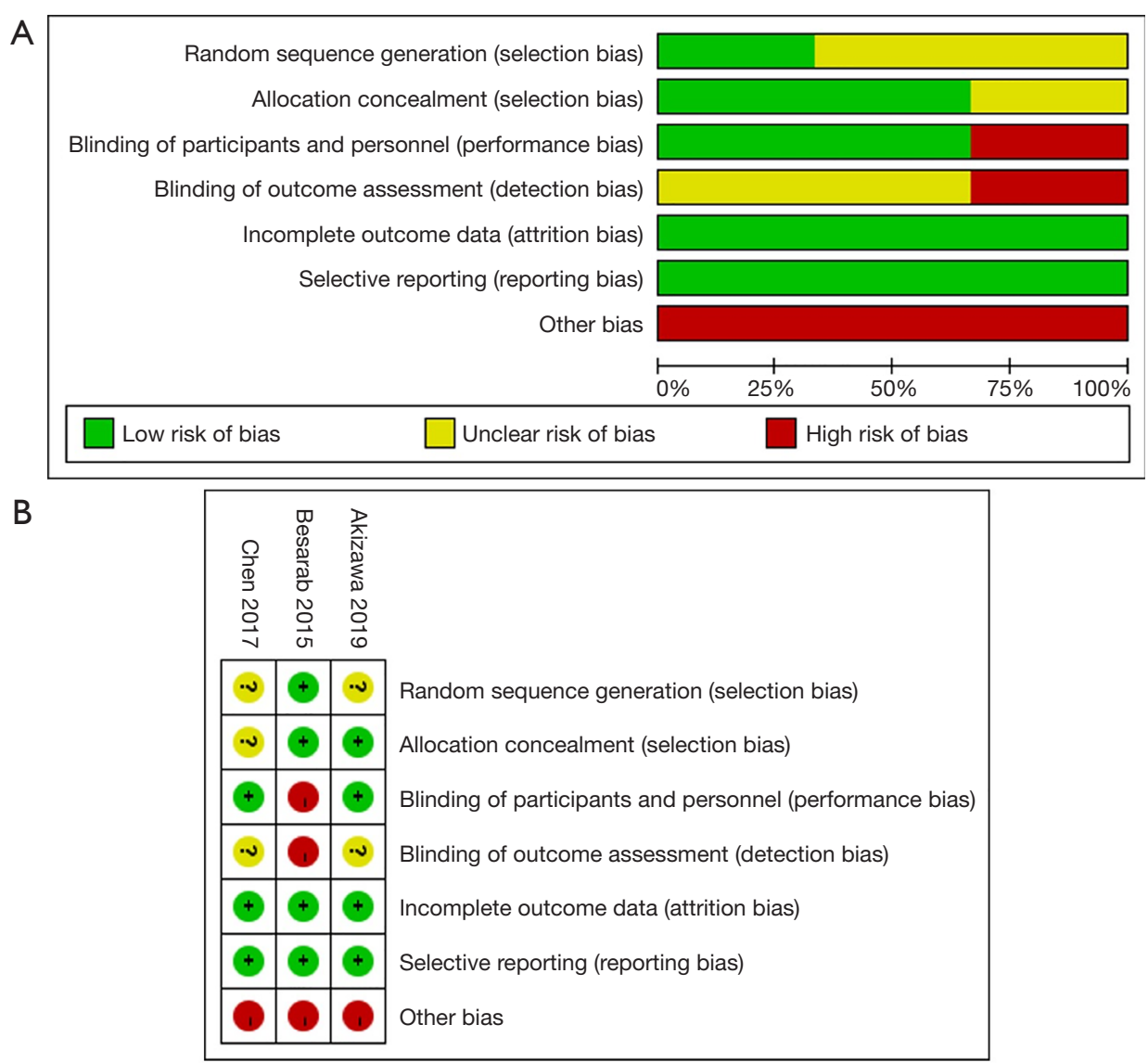

Figure S1 The risk of bias graph and summary. Except for random sequence generation with low risk of $33.3 \%$, allocation concealment with low risk of $66.7 \%$, blinding of personnel with low risk of $66.7 \%$ and blinding of outcome assessment with low risk of $0 \%$, other items were assessed as $100 \%$ of high risk (A). Akizawa et al.'s study was graded as lowest risk, while Besarab et al.'s study was with the highest risk (B). 